\title{
Growth dynamics of fourwing saltbush as affected by dif- ferent grazing management systems
}

\author{
DAVID L. PRICE, GARY B. DONART, AND G. MORRIS SOUTHWARD
}

\section{Abstract}

Individual leaders of fourwing saltbush were permanently marked and their growth responses monitored during a 3-year study in a shortduration grazing system, a 4-pasture rotation system, and in ungrazed exclosures. Primary and secondary leader growth and numbers of secondaries were responses of interest. Plants continuously browsed by cattle were usually maintained in a hedged form and produced relatively little growth. There was little difference in growth responses between plants in the 4-pasture rotation and the shortduration system when the shortduration rotation cycle was 32 days. However, when the rotation cycle was increased to 64 days, there was a substantial increase of growth for plants in the shortduration system. Plants protected from browsing for 1 year also responded with progressively less leader production as length of protection time increased. We suggest fourwing saltbush plants respond to $\$ 60$-day deferment at the beginning of the growing season.

\section{Key Words: Atriplex canescens, utilization, rest periods}

There is an increasing interest in the use of intensively managed grazing systems on private and public rangelands. This interest arises primarily from the need of ranchers for an increased net return on the invested dollar while maintaining rangeland resources. Quantifying the effects of different grazing management systems on individual plant responses can help define and refine basic ideas behind intensive grazing management.

Fourwing saltbush (Atriplex canescens [Pursh.] Nutt.), a common species on New Mexico rangelands, was monitored in the study. Fourwing can be an important browse species on mountainshrub and desert-shrub regions of New Mexico, especially during the dormant period for herbaceous vegetation.

Many clipping studies have been designed to simulate intensity, frequency, and season of defoliation by herbivores. These studies can suggest guidelines for grazing systems, but several differences exist between the effects of clipping and grazing on plants (Culley et al. 1933, Jameson 1963). However, there are limitations using grazing animals because good control is not always possible.

Authors are former graduate research assistant and professor, Department of Animal and Range Sciences, and professor, Department of Experimental Statistics, New Mexico State University, Las Cruces 88003.

This study was conducted in cooperation with the Bureau of Land Management and published as Journal article No. 1365, Agricultural Experiment Station, New Mexico State University, Las Cruces 88003.

Manuscript accepted 31 October 1988.
Undesirable effects of herbage removal on aboveground parts of shrubs are generally in proportion to intensity of clipping or browsing (Bruce 1956, Marshall et al. 1955, Maggs 1959, Shepard 1971). Aldous (1952), Garrison (1953), and Young and Payne (1948) found proper use of shrubs varied from 20 to $75 \%$, depending on species. DeBano (1957), Garrison (1953), and Shepard (1971) reported increases in lateral branching after removal of current annual twig growth from sagebrush and bitterbush. These workers indicate production by individual browse plants can be increased by defoliation if intensity of defoliation is not excessive. Buwai and Trlica (1977) and Trlica et al. (1977) found both detrimental and stimulating effects of removal on several shrubs, depending on intensity and phenological stages of the plant. Davis (1953) also found positive effects of proper defoliation decline after several years if the treatments are not continued. Pieper and Donart (1978) commented that few studies have been done on effects of defoliation on fourwing saltbush. However, these studies indicate fourwing saltbush responds similarly to other shrub species, depending on intensity and frequency of defoliation and phenological stage of the plant at time of defoliation (Buwai and Trlica 1977, Nord and Stalling 1976, Trlica et al. 1977).

In New Mexico, vigor and productive capacity of fourwing plants is usually reduced under a system of year-long grazing, and plants occurring in moderate to low densities in grassland areas need 1 season of rest every 3 or 4 years to maintain populations (Pieper and Donart 1978).

The primary objective of this study was to quantify the growth response of fourwing saltbush to several sequences of rest, deferment and browsing affected by a series of exclosures and grazing systems.

\section{Study Area}

The study was conducted on the Fort Stanton Experimental Ranch, comprising about 10,600 ha in Lincoln County, southcentral New Mexico. Elevation varies from 1,950 to 2,250 m, with a general topography of level mesas, steep canyons, and rolling hills. Mean minimum temperature is $-6.7^{\circ} \mathrm{C}$ in January, mean maximum is $28.8^{\circ} \mathrm{C}$ in July, and mean annual temperature is $11.1^{\circ} \mathrm{C}$ (Groce and Pieper 1967, Pieper et al. 1978). The frost-free period is approximately 160 days from early May until the middle of October (Lowance 1967). Mean annual precipitation for 1876 to 1982 was about $380 \mathrm{~mm}$ (USDC, NOAA 1982) with $60 \%$ occurring during the growing season (Pieper and Donart 1978). Monthly and 
Table 1. Monthly and total precipitation (cm) in 1981, 1982 and 1983 and 78 year average.

\begin{tabular}{lcccc}
\hline \hline Month & 1981 & 1982 & 1983 & $\begin{array}{r}78 \text { year } \\
\text { average }\end{array}$ \\
\hline January & $T$ & 1.3 & 0.7 & 1.5 \\
February & 0.6 & 0.2 & 2.0 & 1.2 \\
March & $\mathrm{T}$ & 0.6 & 2.3 & 1.5 \\
April & 0.9 & - & 1.8 & 1.4 \\
May & 8.2 & 0.3 & 2.0 & 2.3 \\
June & 5.3 & - & 1.2 & 3.2 \\
July & 4.4 & 8.1 & 5.2 & 7.9 \\
August & 8.7 & 6.8 & 4.8 & 6.9 \\
September & 9.2 & 6.9 & 8.8 & 4.7 \\
October & 2.1 & 6.0 & 7.0 & 1.9 \\
November & 1.2 & 1.3 & 5.5 & 0.7 \\
December & - & 2.1 & 0.8 & 1.8 \\
Total & 40.7 & 33.6 & 42.1 & 35.0 \\
\hline
\end{tabular}

total precipitation during the study, 1981 through 1983 , is given in Table 1.

Fort Stanton lies in an ecological transition zone between the juniper-pinyon woodland and the grama-galleta steppe vegetation types (Kuchler 1964). Blue grama (Bouteloua gracilis [H.B.K.] Lag.) is dominant or co-dominant on the mesas and canyon bottoms, while pinyon (Pinus edulis Engelm.), juniper (Juniperus spp.), and wavy leaf oak (Quercus undulata Torr.) are dominants or co-dominants on canyon sides and at higher elevations (Pieper et al. 1971).

\section{Methods}

Management System I was a 7-paddock, short-duration grazing cell that was completed in 1980 . Stocking rate was 13 ha/AUY during the study, 1981-1983. Length of rotation cycles in 1981 and 1982 was 32 days during periods of rapid plant growth (June to August), and 64 days for periods of slow growth or dormancy.
However, there was no period of rapid growth in 1983 because of late summer rains. Consequently, the rotation cycle was maintained at 64 days throughout the year.

Management System II was a 4-pasture (100 ha each) rotation grazing system initiated in 1969 (Pfister et al. 1984). Stocking rate was $15 \mathrm{ha} / \mathrm{AUY}$ in 1981, but was reduced to $22 \mathrm{ha} / \mathrm{AUY}$ in 1982 and 1983 for management reasons.

In Management System I, the short-duration grazing system, 1 paddock ( $139 \mathrm{ha}$ ) was chosen for observation of fourwing saltbush. One group of 10 plants was included in a 0.8 -ha deer- and cattleproof exclosure erected in 1969 and designated as the 20-year rest treatment (20-YR). A second group of 10 plants was included in a cattle-proof exclosure built in 1972 adjacent to the deer exclosure. The exclosure was removed in 1981 and plants were subjected to short-duration grazing during this study. Plants from the group were designated as the shortduration paddock 8 treatment (SD-8). Adjacent to the removed cattle exposure, a third group of 10 fourwing saltbush plant was chosen. These plants had been subjected to year-long grazing from 1969 to 1980 (Pieper and Donart 1978), and then short-duration grazing from 1980 throughout this study. This group was designated as the short-duration paddock 7 treatment (SD-7). Stock density in SD-7 and SD-8 was 0.5 AU/ha.

In Management System II, the 4-pasture rotation grazing system, only 2 of the 4 pastures were used. One group of 10 fourwing plants was chosen in each of the 2 pastures. One pasture was rested from February 1981 through February 1982, and grazed the remainder of the study. The other pasture was grazed continuously from February 1980 through February 1983, and rested until February 1984. Stock density in both pastures was $0.09 \mathrm{AU} / \mathrm{ha}$ in 1981 and $0.06 \mathrm{AU} / \mathrm{ha}$ in 1982-1983. In March 1982, one more group of 10 plants was chosen in each of the same 2 pastures. These were protected from cattle browsing to achieve the desired sequences and lengths of rest. For Management System II, treatment designations were assigned by year of the study (e.g., 1-year rest treatment [1-YR], 2-year rest treatment [2-YR], 3-yr rest [3-YR],

$1981 \quad 1982 \quad 1983$

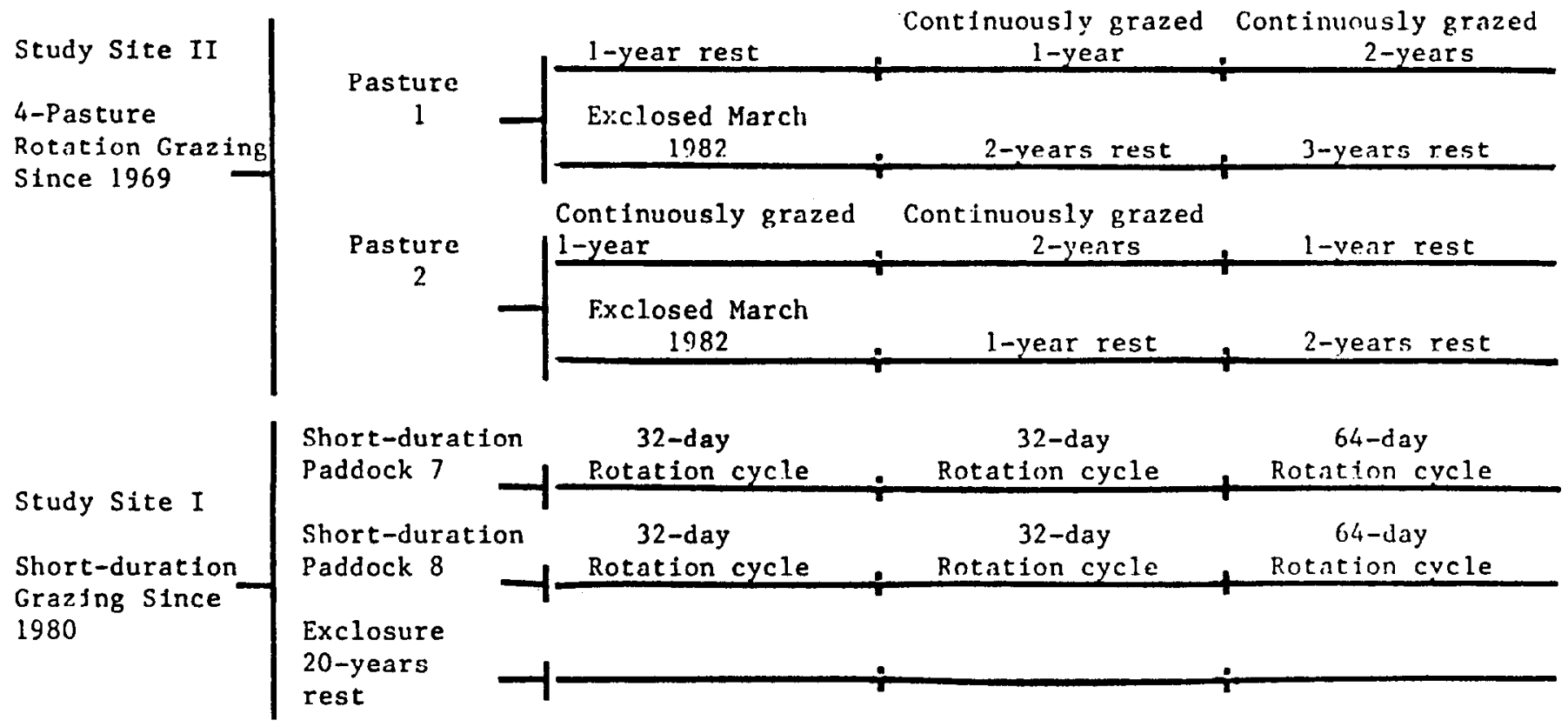

Fis. 1. Treatment structure for fourwing saltbush study during growing seasons of 1981 through 1983. 
continuous-grazing, 1-year [CG-1] and continuous-grazing, 2-year [CG-2] (Fig. 1).

Sixteen primary leaders on all identified fourwing plants were marked at their base with acrylic paint during the second week of May each year. Growth measurements were taken every 12 to 15 days throughout the growing season until early October. Measurements included primary leader growth, numbers of secondary leaders per primary leader, and total secondary leader growth per primary leader.

Positive increments in growth were determined for each measurement date and summed over each growing season. Data were first analyzed within year using a completely randomized design with plants as replications. Whole model analysis indicated measurement date by treatment interaction terms were significant $(P \leq 0.001)$ for each of the 3 years. Therefore, data were analyzed within year and measurement date. Where $F$ tests were significant $(P<0.05)$, the LSD procedure was used for mean separations at the 95\% level.

\section{Results and Discussion}

\section{Growth Dynamics - 1981}

By late July, primary leader growth of fourwing saltbush was significantly greater in the 1-YR treatment (Table 2). Little additional growth was contributed by primary leaders after July, and

Table 2. Cumulative mean growth (cm) by measurement date of primary leader length, secondary leader length per primary, numbers of secondary leaders per primary and total length (primary plus secondary length) for fourwing saltbush, 1981 through 1983.

\begin{tabular}{|c|c|c|c|c|c|c|c|c|c|c|c|c|}
\hline \multirow{2}{*}{$\begin{array}{l}\text { Date/ } \\
\text { treatment }\end{array}$} & \multicolumn{3}{|c|}{ Mean primary length } & \multicolumn{3}{|c|}{ Mean secondary length } & \multicolumn{3}{|c|}{ Mean numbers of secondaries } & \multicolumn{3}{|c|}{ Mean total length } \\
\hline & 1981 & 1982 & 1983 & 1981 & 1982 & 1983 & 1981 & 1982 & 1983 & 1981 & 1982 & 1983 \\
\hline \multicolumn{13}{|l|}{ Mid-May } \\
\hline $\begin{array}{l}1-Y^{1} \\
2-Y R \\
\text { 3-YR } \\
20-Y R \\
\text { SD-7 } \\
\text { SD-8 } \\
\text { CG-1 } \\
\text { CG-2 }\end{array}$ & $\begin{array}{l}3.8 \mathrm{a}^{2} \\
-3 \\
-\overline{2.4 b c} \\
2.0 \mathrm{c} \\
2.0 \mathrm{c} \\
3.0 \mathrm{~b} \\
-\end{array}$ & $\begin{array}{l}2.5 \mathrm{a} \\
2.0 \mathrm{a} \\
1 . \overline{b c} \\
1.1 \mathrm{bc} \\
0.9 \mathrm{c} \\
1.2 \mathrm{bc} \\
1.4 \mathrm{~b}\end{array}$ & $\begin{array}{l}8.2 \mathrm{a} \\
4.6 \mathrm{~cd} \\
5.2 \mathrm{bc} \\
3.5 \mathrm{de} \\
6.1 \mathrm{~b} \\
6.3 \mathrm{~b} \\
-\bar{c} \\
3.1 \mathrm{e}\end{array}$ & & & & & & & & & \\
\hline \multicolumn{13}{|l|}{ Early June } \\
\hline $\begin{array}{l}\text { 1-YR } \\
\text { 2-YR } \\
\text { 3-YR } \\
20-Y R \\
\text { SD-7 } \\
\text { SD-8 } \\
\text { CG-1 } \\
\text { CG-2 }\end{array}$ & $\begin{array}{l}8.1 \mathrm{a} \\
- \\
\overline{-} \\
4.3 \mathrm{~cd} \\
6.1 \mathrm{~b} \\
5.5 \mathrm{bc} \\
3.7 \mathrm{~d} \\
-\end{array}$ & $\begin{array}{l}4.1 \mathrm{a} \\
2.5 \mathrm{~b} \\
\overline{1.9 b} \\
1.8 \mathrm{~b} \\
2.1 \mathrm{~b} \\
1.7 \mathrm{~b} \\
2.1 \mathrm{~b}\end{array}$ & $\begin{array}{c}12.5 \mathrm{a} \\
7.5 \mathrm{c} \\
8.3 \mathrm{c} \\
5.5 \mathrm{~d} \\
10.9 \mathrm{ab} \\
10.7 \mathrm{~b} \\
\overline{4} .1 \mathrm{~d}\end{array}$ & & & & & & & & & \\
\hline \multicolumn{13}{|l|}{ Late June } \\
\hline $\begin{array}{l}\text { 1-YR } \\
\text { 2-YR } \\
\text { 3-YR } \\
20-Y R \\
\text { SD-7 } \\
\text { SD-8 } \\
\text { CG-1 } \\
\text { CG-2 }\end{array}$ & $\begin{array}{c}13.2 \mathrm{a} \\
- \\
-\overline{-} \\
5.4 \mathrm{c} \\
6.6 \mathrm{~b} \\
6.2 \mathrm{~b} \\
4.3 \mathrm{c} \\
-\end{array}$ & $\begin{array}{l}9.8 \mathrm{a} \\
2.5 \mathrm{~cd} \\
3 \overline{-} \\
3.3 \mathrm{c} \\
3.4 \mathrm{c} \\
6.4 \mathrm{~b} \\
1.7 \mathrm{~d} \\
2.8 \mathrm{~cd}\end{array}$ & $\begin{array}{c}17.5 \mathrm{a} \\
11.1 \mathrm{~b} \\
10.8 \mathrm{~b} \\
7.4 \mathrm{c} \\
17.8 \mathrm{a} \\
16.9 \mathrm{a} \\
0 \\
5.0 \mathrm{c}\end{array}$ & $\begin{array}{c}3.1 \mathrm{a} \\
- \\
-\overline{0.1 \mathrm{~b}} \\
0.0 \mathrm{~b} \\
0.0 \mathrm{~b} \\
0.1 \mathrm{~b} \\
-\end{array}$ & $\begin{array}{l}3.0 \mathrm{a} \\
0.0 \mathrm{~b} \\
- \\
0.4 \mathrm{~b} \\
0.2 \mathrm{~b} \\
3.0 \mathrm{a} \\
0.0 \mathrm{~b} \\
0.2 \mathrm{~b}\end{array}$ & $\begin{array}{l}1.5 \mathrm{c} \\
0.7 \mathrm{c} \\
2.9 \mathrm{bc} \\
0.4 \mathrm{c} \\
6.0 \mathrm{a} \\
4.8 \mathrm{ab} \\
\overline{0.5 \mathrm{c}}\end{array}$ & $\begin{array}{c}2.3 \mathrm{a} \\
- \\
\overline{0.1 \mathrm{~b}} \\
0.0 \mathrm{~b} \\
0.0 \mathrm{~b} \\
0.1 \mathrm{~b} \\
-\end{array}$ & $\begin{array}{l}1.2 \mathrm{c} \\
0.0 \mathrm{~b} \\
\overline{0.2 \mathrm{~b}} \\
0.1 \mathrm{~b} \\
1.1 \mathrm{a} \\
0.0 \mathrm{~b} \\
0.2 \mathrm{~b}\end{array}$ & $\begin{array}{l}0.8 \mathrm{bc} \\
0.3 \mathrm{c} \\
1.7 \mathrm{ab} \\
0.2 \mathrm{c} \\
1.9 \mathrm{a} \\
1.7 \mathrm{ab} \\
\overline{0} \\
0.3 \mathrm{c}\end{array}$ & $\begin{array}{l}16.3 \mathrm{a} \\
- \\
\overline{5.5 b c} \\
6.6 \mathrm{~b} \\
6.2 \mathrm{bc} \\
4.4 \mathrm{c} \\
-\end{array}$ & $\begin{array}{c}12.8 \mathrm{a} \\
2.5 \mathrm{c} \\
3 . \bar{c} \\
3.6 \mathrm{c} \\
9.4 \mathrm{~b} \\
1.7 \mathrm{c} \\
3.0 \mathrm{c}\end{array}$ & $\begin{array}{c}19.0 \mathrm{~b} \\
11.8 \mathrm{~cd} \\
13.7 \mathrm{c} \\
7.8 \mathrm{de} \\
23.8 \mathrm{a} \\
21.7 \mathrm{ab} \\
\overline{5} \\
5.5 \mathrm{e}\end{array}$ \\
\hline \multicolumn{13}{|l|}{ Early July } \\
\hline $\begin{array}{l}\text { 1-YR } \\
\text { 2-YR } \\
\text { 3-YR } \\
20-Y R \\
\text { SD-7 } \\
\text { SD-8 } \\
\text { CG-1 } \\
\text { CG-2 }\end{array}$ & $\begin{array}{c}15.5 \mathrm{a} \\
- \\
5.9 \mathrm{c} \\
8.3 \mathrm{~b} \\
8.5 \mathrm{~b} \\
5.0 \mathrm{c} \\
-\end{array}$ & $\begin{array}{r}13.5 \mathrm{a} \\
9.2 \mathrm{~b} \\
\overline{-} \\
4.7 \mathrm{c} \\
5.7 \mathrm{c} \\
12.5 \mathrm{a} \\
5.0 \mathrm{c} \\
3.7 \mathrm{c}\end{array}$ & $\begin{array}{l}20.8 \mathrm{a} \\
13.2 \mathrm{c} \\
11.9 \mathrm{~cd} \\
8.8 \mathrm{de} \\
18.0 \mathrm{ab} \\
17.1 \mathrm{~b} \\
\overline{-} \\
5.6 \mathrm{~b}\end{array}$ & $\begin{array}{c}6.0 \mathrm{a} \\
- \\
-\overline{1.1 \mathrm{~b}} \\
2.6 \mathrm{~b} \\
2.2 \mathrm{~b} \\
1.0 \mathrm{~b} \\
-\end{array}$ & $\begin{array}{l}8.7 \mathrm{a} \\
0.1 \mathrm{~b} \\
\overline{1.3 \mathrm{~b}} \\
1.5 \mathrm{~b} \\
8.8 \mathrm{a} \\
0.2 \mathrm{~b} \\
1.3 \mathrm{~b}\end{array}$ & $\begin{array}{l}5.6 \mathrm{abc} \\
2.4 \mathrm{~d} \\
3.8 \mathrm{~cd} \\
1.8 \mathrm{~d} \\
7.6 \mathrm{a} \\
6.0 \mathrm{~b} \\
\overline{2.7 \mathrm{bcd}}\end{array}$ & $\begin{array}{c}2.6 \mathrm{a} \\
- \\
-\overline{0.6 b} \\
1.0 \mathrm{~b} \\
0.9 \mathrm{~b} \\
0.4 \mathrm{~b} \\
-\end{array}$ & $\begin{array}{l}2.0 \mathrm{a} \\
0.1 \mathrm{~b} \\
\overline{0.6 \mathrm{~b}} \\
0.7 \mathrm{~b} \\
2.7 \mathrm{a} \\
0.1 \mathrm{~b} \\
0.7 \mathrm{~b}\end{array}$ & $\begin{array}{l}2.5 \mathrm{ab} \\
0.1 \mathrm{c} \\
2.2 \mathrm{abc} \\
0.8 \mathrm{~d} \\
2.8 \mathrm{a} \\
2.3 \mathrm{abc} \\
\overline{1} \\
1.2 \mathrm{bcd}\end{array}$ & $\begin{array}{r}21.5 \mathrm{a} \\
- \\
7.0 \mathrm{c} \\
10.9 \mathrm{~b} \\
10.7 \mathrm{~b} \\
6.0 \mathrm{c} \\
-\end{array}$ & $\begin{array}{r}22.2 \mathrm{a} \\
9.3 \mathrm{~b} \\
\overline{6.0 \mathrm{~b}} \\
7.2 \mathrm{~b} \\
21.3 \mathrm{a} \\
5.2 \mathrm{~b} \\
5.0 \mathrm{~b}\end{array}$ & $\begin{array}{l}26.4 \mathrm{a} \\
15.6 \mathrm{~b} \\
15.7 \mathrm{~b} \\
10.6 \mathrm{bc} \\
25.6 \mathrm{a} \\
23.1 \mathrm{a} \\
\overline{8.3 \mathrm{c}}\end{array}$ \\
\hline \multicolumn{13}{|l|}{ Late July } \\
\hline $\begin{array}{l}\text { 1-YR } \\
2-Y R \\
\text { 3-YR } \\
20-Y R \\
\text { SD-7 } \\
\text { SD-8 } \\
\text { CG-1 } \\
\text { CG-2 }\end{array}$ & $\begin{array}{c}19.0 \mathrm{a} \\
- \\
-\overline{6.4 c} \\
8.8 \mathrm{~b} \\
9.2 \mathrm{~b} \\
5.5 \mathrm{c} \\
-\end{array}$ & $\begin{array}{c}15.8 \mathrm{ab} \\
17.9 \mathrm{a} \\
-\overline{-2 \mathrm{c}} \\
5.7 \mathrm{c} \\
13.4 \mathrm{~b} \\
6.4 \mathrm{c} \\
4.6 \mathrm{c}\end{array}$ & $\begin{array}{c}22.7 \mathrm{a} \\
14 . \mathrm{acd} \\
12.1 \mathrm{de} \\
9.2 \mathrm{ef} \\
18.5 \mathrm{~b} \\
17.5 \mathrm{bc} \\
\overline{6.1 \mathrm{f}}\end{array}$ & $\begin{array}{c}14.6 \mathrm{a} \\
- \\
\overline{1} \\
1.9 \mathrm{~b} \\
4.6 \mathrm{~b} \\
4.0 \mathrm{~b} \\
2.1 \mathrm{~b} \\
-\end{array}$ & $\begin{array}{c}12.9 \mathrm{a} \\
1.9 \mathrm{~b} \\
- \\
1.6 \mathrm{~b} \\
2.2 \mathrm{~b} \\
10.3 \mathrm{a} \\
1.8 \mathrm{~b} \\
2.0 \mathrm{~b}\end{array}$ & $\begin{array}{c}9.2 \mathrm{a} \\
3.5 \mathrm{~b} \\
3.8 \mathrm{~b} \\
2.0 \mathrm{~b} \\
12.2 \mathrm{a} \\
9.7 \mathrm{a} \\
0 \\
4.0 \mathrm{~b}\end{array}$ & $\begin{array}{c}3.6 \mathrm{a} \\
- \\
\overline{-} \\
0.9 \mathrm{~b} \\
1.4 \mathrm{~b} \\
1.4 \mathrm{~b} \\
0.9 \mathrm{~b} \\
0\end{array}$ & $\begin{array}{l}2.4 \mathrm{a} \\
0.6 \mathrm{~b} \\
- \\
0.7 \mathrm{~b} \\
0.9 \mathrm{~b} \\
3.2 \mathrm{a} \\
0.6 \mathrm{~b} \\
0.9 \mathrm{~b}\end{array}$ & $\begin{array}{l}3.3 \mathrm{a} \\
1.4 \mathrm{c} \\
2.2 \mathrm{abc} \\
0.9 \mathrm{c} \\
3.6 \mathrm{a} \\
3.0 \mathrm{ab} \\
\overline{-8 \mathrm{bc}}\end{array}$ & $\begin{array}{c}33.6 \mathrm{a} \\
- \\
\overline{-} \\
8.3 \mathrm{bc} \\
13.4 \mathrm{~b} \\
13.2 \mathrm{bc} \\
7.6 \mathrm{c} \\
-\end{array}$ & $\begin{array}{c}28.7 \mathrm{a} \\
19.8 \mathrm{~b} \\
- \\
6.8 \mathrm{c} \\
8.9 \mathrm{c} \\
23.7 \mathrm{ab} \\
8.2 \mathrm{c} \\
6.6 \mathrm{c}\end{array}$ & $\begin{array}{l}31.9 \mathrm{a} \\
17.6 \mathrm{~b} \\
15.9 \mathrm{bc} \\
11.2 \mathrm{bc} \\
30.7 \mathrm{a} \\
27.2 \mathrm{a} \\
\overline{-} \\
10.1 \mathrm{c}\end{array}$ \\
\hline
\end{tabular}


Table 2. Continued.

\begin{tabular}{|c|c|c|c|c|c|c|c|c|c|c|c|c|}
\hline \multirow{2}{*}{$\begin{array}{l}\text { Date/ } \\
\text { treatment }\end{array}$} & \multicolumn{3}{|c|}{ Mean primary length } & \multicolumn{3}{|c|}{ Mean secondary length } & \multicolumn{3}{|c|}{ Mean numbers of secondaries } & \multicolumn{3}{|c|}{ Mean total length } \\
\hline & 1981 & 1982 & 1983 & 1981 & 1982 & 1983 & 1981 & 1982 & 1983 & 1981 & 1982 & 1983 \\
\hline \multicolumn{13}{|c|}{ Mid-August } \\
\hline $\begin{array}{l}\text { 1-YR } \\
2-Y R \\
3-Y R \\
20-Y R \\
\text { SD-7 } \\
\text { SD-8 } \\
\text { CG-1 } \\
\text { CG-2 }\end{array}$ & $\begin{array}{c}21.9 \mathrm{a} \\
- \\
-\overline{6.6 c} \\
9.6 \mathrm{~b} \\
9.8 \mathrm{~b} \\
6.6 \mathrm{c} \\
-\end{array}$ & $\begin{array}{c}17.7 \mathrm{ab} \\
21.2 \mathrm{a} \\
-\overline{5.8 \mathrm{c}} \\
7.3 \mathrm{c} \\
14.3 \mathrm{~b} \\
6.7 \mathrm{c} \\
5.1 \mathrm{c}\end{array}$ & $\begin{array}{c}23.7 \mathrm{a} \\
14.7 \mathrm{~cd} \\
12.9 \mathrm{de} \\
9.5 \mathrm{ef} \\
19.4 \mathrm{~b} \\
18.1 \mathrm{c} \\
-\overline{6.3 \mathrm{f}}\end{array}$ & $\begin{array}{c}23.2 \mathrm{a} \\
- \\
- \\
3.4 \mathrm{~b} \\
8.1 \mathrm{~b} \\
6.1 \mathrm{~b} \\
3.8 \mathrm{~b} \\
-\end{array}$ & $\begin{array}{r}16.6 \mathrm{a} \\
2.8 \mathrm{~b} \\
-\overline{2.0 \mathrm{~b}} \\
3.0 \mathrm{~b} \\
11.9 \mathrm{a} \\
2.6 \mathrm{~b} \\
2.9 \mathrm{~b}\end{array}$ & $\begin{array}{c}11.6 \mathrm{bc} \\
3.9 \mathrm{~d} \\
4.9 \mathrm{~d} \\
3.4 \mathrm{~d} \\
19.4 \mathrm{a} \\
14.9 \mathrm{ab} \\
-\overline{\mathrm{c}} \\
5.2 \mathrm{~cd}\end{array}$ & $\begin{array}{c}4.9 \mathrm{a} \\
- \\
-\overline{1 b} \\
2.1 \mathrm{~b} \\
2.3 \mathrm{~b} \\
2.0 \mathrm{~b} \\
1.8 \mathrm{~b} \\
-\end{array}$ & $\begin{array}{c}2.7 \mathrm{a} \\
0.6 \mathrm{~b} \\
-\overline{0.9 b} \\
1.2 \mathrm{~b} \\
3.5 \mathrm{a} \\
0.9 \mathrm{~b} \\
1.3 \mathrm{~b}\end{array}$ & \begin{tabular}{l}
$3.8 \mathrm{a}$ \\
$1.6 \mathrm{c}$ \\
$2.8 \mathrm{abc}$ \\
$1.5 \mathrm{c}$ \\
$4.2 \mathrm{a}$ \\
$3.6 \mathrm{ab}$ \\
\multicolumn{1}{c}{$-\overline{\mathrm{a}}$} \\
$2.1 \mathrm{bc}$
\end{tabular} & $\begin{array}{c}45.1 \mathrm{a} \\
- \\
- \\
10.0 \mathrm{bc} \\
17.7 \mathrm{~b} \\
15.9 \mathrm{bc} \\
9.0 \mathrm{c} \\
-\end{array}$ & $\begin{array}{c}34.4 \mathrm{a} \\
24.0 \mathrm{~b} \\
- \\
7.8 \mathrm{c} \\
10.3 \mathrm{c} \\
26.2 \mathrm{ab} \\
9.3 \mathrm{c} \\
8.0 \mathrm{c}\end{array}$ & $\begin{array}{r}35.3 \mathrm{a} \\
18.6 \mathrm{~b} \\
17.8 \mathrm{~b} \\
12.9 \mathrm{~b} \\
38.8 \mathrm{a} \\
33.0 \mathrm{a} \\
-\overline{\mathrm{a}} \\
11.5 \mathrm{~b}\end{array}$ \\
\hline \multicolumn{13}{|l|}{$\begin{array}{l}\text { Early } \\
\text { October }\end{array}$} \\
\hline $\begin{array}{l}1-Y R \\
2-Y R \\
3-Y R \\
20-Y R \\
\text { SD-7 } \\
\text { SD-8 } \\
\text { CG-1 } \\
\text { CG-2 }\end{array}$ & $\begin{array}{r}22.6 \mathrm{a} \\
- \\
-\overline{6.9 c} \\
9.8 \mathrm{~b} \\
10.1 \mathrm{~b} \\
6.9 \mathrm{c} \\
-\end{array}$ & $\begin{array}{r}18.8 \mathrm{~b} \\
23.2 \mathrm{a} \\
-\overline{7.9 \mathrm{c}} \\
7.8 \mathrm{c} \\
14.9 \mathrm{~b} \\
7.1 \mathrm{c} \\
5.8 \mathrm{c}\end{array}$ & $\begin{array}{c}24.4 \mathrm{a} \\
15.3 \mathrm{~cd} \\
13.3 \mathrm{de} \\
9.8 \mathrm{ef} \\
19.6 \mathrm{~b} \\
18.2 \mathrm{bc} \\
-\overline{7 \mathrm{f}}\end{array}$ & $\begin{array}{c}25.4 \mathrm{a} \\
- \\
-\overline{3.7 b} \\
8.2 \mathrm{~b} \\
6.2 \mathrm{~b} \\
4.0 \mathrm{~b} \\
-\end{array}$ & $\begin{array}{c}19.2 \mathrm{a} \\
3.3 \mathrm{c} \\
- \\
6.4 \mathrm{bc} \\
3.5 \mathrm{c} \\
13.0 \mathrm{ab} \\
3.5 \mathrm{c} \\
3.5 \mathrm{c}\end{array}$ & $\begin{array}{r}18.1 \mathrm{a} \\
4.1 \mathrm{~b} \\
5.0 \mathrm{~b} \\
3.5 \mathrm{~b} \\
20.4 \mathrm{a} \\
15.7 \mathrm{a} \\
-\overline{5.6 b}\end{array}$ & $\begin{array}{c}5.5 a \\
- \\
-\overline{2.3 b} \\
2.4 b \\
2.1 b \\
2.0 b \\
-\end{array}$ & $\begin{array}{l}3.0 \mathrm{ab} \\
0.8 \mathrm{c} \\
- \\
2.2 \mathrm{bc} \\
1.4 \mathrm{c} \\
3.9 \mathrm{a} \\
1.3 \mathrm{c} \\
1.7 \mathrm{bc}\end{array}$ & $\begin{array}{l}4.0 \mathrm{a} \\
1.6 \mathrm{c} \\
2.8 \mathrm{abc} \\
1.6 \mathrm{c} \\
4.4 \mathrm{a} \\
3.8 \mathrm{ab} \\
\overline{-} \\
2.2 \mathrm{bc}\end{array}$ & $\begin{array}{c}48.0 \mathrm{a} \\
- \\
10.6 \mathrm{~b} \\
19.0 \mathrm{~b} \\
16.3 \mathrm{~b} \\
10.3 \mathrm{~b} \\
-\end{array}$ & $\begin{array}{c}38.0 \mathrm{a} \\
26.5 \mathrm{~b} \\
- \\
14.3 \mathrm{c} \\
11.3 \mathrm{c} \\
27.9 \mathrm{~b} \\
10.6 \mathrm{c} \\
9.3 \mathrm{c}\end{array}$ & $\begin{array}{r}42.5 \mathrm{a} \\
19.4 \mathrm{~b} \\
18.3 \mathrm{~b} \\
13.3 \mathrm{~b} \\
40.0 \mathrm{a} \\
33.9 \mathrm{a} \\
-\overline{12.1 \mathrm{~b}}\end{array}$ \\
\hline
\end{tabular}

11-YR = one year rest; 2-YR = two year rest; 3-YR = three year rest; 20-YR = 20 year rest; SD-7 = short-duration paddock seven; SD-8 = short-duration paddock eight; CG-1 = continuous grazing on year; CG-2 = continuous grazing two years.

${ }^{2}$ Different letters within measurement date and column indicate significant differences $(P \leq 0.05)$.

${ }^{3}$ Dashes ( - ) indicate no data collected.

treatment means separated into 3 groups. The first group included plants with the least growth and occurred on plants in the CG-1 and 20-YR treatments. Plants in the SD-7 and SD-8 treatments produced intermediate primary growth and plants in the 1-YR treatment produced the most primary growth (Table 2).

Initiation of axillary buds that produced secondary leaders began in June in the 1-YR treatment, but plants in remaining treatments did not produce much secondary growth until July. By October, secondary leader growth and numbers of secondaries were greatest for plants in the 1-YR treatment. Secondary growth and numbers were not different for plants between the remaining treatments for any measurement date (Table 2).

From July until October, major contributions to total growth (primary plus secondary growth) was from secondary growth in all treatments, but this response was particularly evident in the 1-YR treatment. Secondary growth was not significantly different among the remaining treatments from July through October; however, fourwing plants in the SD-7 and SD-8 treatments tended to produce more growth than plants in the CG-1 and 20-YR treatments (Table 2).

\section{Growth Dynamics-1982}

As in 1981, little additional growth was contributed by primary leaders after July. Plants in the 1-yr, 2-yr, and SD-8 treatments tended to produce the greatest primary growth while plants in the 20-yr, SD-7 and CG-1 and CG-2 treatments produced substantially lesser amounts of primary growth (Table 2).

Fourwing plants in the SD-8 treatment attained greater primary growth than might be expected as compared to the SD-7 treatment (Table 2). This can be explained because plants in the SD-8 treatment in 1981 were exposed to grazing after removal of the exclosure that had protected them for 9 years. During protection, plants had become decadent with long, brittle upper branches. When exposed to browsing by cattle, upper branches were easily broken at the base, leaving only older and stouter branches with few areas of active growth. However, enough leaders remained intact to maintain sample numbers. New areas of active growth began to appear in 1982, a response caused by breakage of the younger upper branches. These new areas of active growth were well down in and among older branches, making cattle access to them difficult. In 1982, leaders on plants in the SD-8 treatment were unbrowsed until they reached a substantial length and cattle were able to reach them. Fourwing plants in the SD-7 treatment were in a hedged condition because of continuous browsing since 1969 . Therefore, most new leader growth on these plants was vulnerable to browsing as soon as it was a few centimeters long. Secondary leader growth began in June 1982, and plants in the 1-YR and SD-8 treatments produced significantly greater lengths and numbers of secondaries by August (Table 2). Secondary growth was greater than expected in the SD-8 treatment compared to the SD-7 treatment. Again, this was probably a result of position of new growth down in the main branches of the plants where the leaders were not accessible to browsing and more axillary buds were left intact to develop secondary growth.

In terms of total growth, plants in the 1-YR treatment produced significantly more than plants in the 2-YR and SD-8 treatments and these, in turn, produced more than plants in the remaining treatments (Table 2). These data indicate a trend of reduced vegetative growth on a per leader basis for fourwing plants that are protected from browsing for more than 1 year (Davis 1953).

\section{Growth Dynamics-1983}

Because of late summer rains in 1983, the rotation cycle for the short duration grazing system (Management System I) was maintained at 64 days through the growing season rather than 32 days as in 1981 and 1982 (Fig. 1). This decision had a significant impact on study results.

By late June 1984, primary leader growth for fourwing in the 1-YR, SD-7 and SD-8 treatments was significantly greater than leader growth for the 2-YR and 3-YR treatments (Table 2). Plants in the 20-YR and CG-2 treatments produced the least amount of growth. This trend in early-season growth was maintained through the end of the growing season.

Secondary leader growth began in June and by October, secondary length was significantly greater in the 1-YR, SD-7, and SD-8 treatments (Table 2). Numbers of secondaries produced also fol- 
lowed this same trend; however there was a lack of separation among treatment means.

Total growth in June 1983 was greatest for plants in the 1-YR, SD-7 and SD-8 treatments, intermediate in the 2-YR and 3-YR treatments, and least in the 20-YR and CG-2 treatments (Table 2). This relationship was the same at the end of the growing season. However, means for the 2-YR, 3-YR, 20-YR, and CG-2 treatments were not statistically different.

An important point with respect to the data is that plants in the SD-7 and SD-8 treatments were subjected to 2 heavy defoliations during the 1983 growing season, but they still produced as much total growth on a per leader basis as plants in the 1-YR treatment. Because of the 64-day rotation cycle, fourwing plants in the SD-7 and SD-8 treatments received their first defoliation about 60 days after spring growth started. This 60 -day deferment period allowed primary leaders to reach an average length of $11 \mathrm{~cm}$ before being browsed (Table 2). From general field observations, it was noted that leaders 10 to $15 \mathrm{~cm}$ long became slightly woody at their bases compared to shorter leaders, but they remained flexible compared to longer and older leaders. When cattle browsed plants in the SD-7 and SD-8 treatments, they removed terminal buds and younger tissue at the ends of leaders, leaving the woody portion of the leader intact. Therefore, axillary buds were left intact on plants, but the most rapidly growing tissue and terminal buds were removed. This probably reduced inhibition on axillary buds (Jameson 1963). However, if fourwing plants were browsed before leaders became woody at their bases, the entire leader was generally pulled off leaving very few axillary buds intact. This is what generally took place in the continuously grazed treatments (CG-1 or CG-2) and in the SD-7 and SD-8 treatments when the rotation cycle was too short (32-day rotation) to allow primary leaders to reach optimum length.

Conversely, if fourwing plants were rested 1 complete year, leaders became very long, woody, and brittle. When cattle browsed the plants the next year, these fragile leaders were easily broken off, thereby causing severe plant damage. This type of damage was noted twice during the study, once in 1981 in the SD-8 treatment when plants were browsed after 9 years protection and again in 1982 in the CG-1 treatment after plants had been rested in 1981 (Fig. 1). In addition, fourwing plants rested more than 1 year began to decline in growth of primary and secondary leaders and numbers of secondary leaders (Table 2). These data support findings of Davis (1953) and Buwai and Trlica (1977).

\section{Summary and Conclusions}

Results of this study indicate continuous browsing of fourwing saltbush plants produced relatively little leader growth. A high percentage of primary leaders produced secondary leaders, but the plants were browsed so intensively and frequently that only a few secondary leaders developed on each primary. Secondaries were likewise browsed before much growth was producod.

Fourwing plants browsed under proper intensity and frequency produced as much leader growth as plants rested 1 complete year. The 60-day deferment after onset of spring growth, combined with periodic rest thereafter, allowed primary leaders to attain enough growth so cattle did not browse too intensively. Therefore, a greater number of axillary buds were left intact and produced secondary leaders of substantial length.

Fourwing plants rested for 1, 2 and 3 or more years produced progressively more flower stalks (Price 1985) and progressively less leader growth. Therefore, proper sequences and lengths of rest maintain the plant in a more productive vegetative state. Even continuously browsed plants produced as much leader growth as plants rested too long (20-YR).

In conclusion, browsing can stimulate growth of fourwing salt- bush if the proper rest sequence is used. A short-duration grazing system can help achieve proper rest, but is not necessary. The key is to allow enough deferment in the early growing season. Any grazing strategy which provides at least 60 days deferment in the early growing season will allow for adequate growth.

\section{Literature Cited}

Aldous, S.E. 1952. Deer browse clipping study in the Lake states Region. J. Wildl. Manage. 16:401-409.

Bruce, D. 1956. Effect of defoliation on long leaf pine seedlings. Forestry 38:649-650.

Buwai, M., and M.J. Trlica. 1977. Multiple defoliation effects on herbage yield, vigor and total nonstructural carbohydrates of five range species. J. Range Manage. 30:164-171.

Culley, M.J., R.S. Campbell, and R.H. Canfield. 1933. Values and limitations of clipped quadrats. Ecology 14:35-39.

Davis, C.W. 1953. Clipped plots on Annie Creek and Hyster Experimental areas for 1952. South Dakota Dept. Game, Fish and Parks P-R Project 12-R-10. Completion Report.

DeBano, L.F. 1957. The effects of season and degree of use on the physiological response and nutritive content of desert forage plants. M.S. Thesis. Utah State Univ. In: R.E. Sosebee (Ed.). Rangeland Plant Physiology. Soc. Range Manage. Range Sci. Series No. 4.

Garrison, G.A. 1953. Effects of clipping on some range shrubs. J. Range Manage. 6:309-317.

Groce, V.L., and R.D. Pieper. 1967. Cover, herbage production and botanical composition on foothill range sites in Southcentral New Mexico. New Mexico Agr. Exp. Sta. Res. Rep. 128.

Jameson, D.A. 1963. Evaluation of the responses of individual plants to grazing, p. 109-116. In: Range Research Methods. USDA Misc. Pub. 940.172 p.

Kuchler, A.W. 1964. Potential natural vegetation of the conterminus United States. American Geog. Soc., New York.

Lowance, S.A. 1967. Burning and fertilization of blue grama range site. M.S. Thesis. New Mexico State Univ., Las Cruces.

Magsa, D.H. 1959. The pruning response of one-year-old apple trees. Ann. Bot. 23:319-330.

Marshall, W.H., T. Schantz-Haneen, and K.E. Singness. 1955. Effects of simulated overbrowsing on small red and white pine trees. J. Forestry 53:420-424.

Pfister, J.A., G.B. Donart, R.D. Pieper, J.D. Wallace, and E.E. Parker. 1984. Cattle diets under continuous and four-pasture, one-herd grazing systems in southcentral New Mexico. J. Range Manage. 37:50-54.

Pieper, R.D., and G.B. Donart. 1978. Reponse of fourwing saltbush to periods of protection. J. Range Manage. 31:314-315.

Pieper, R.D., G.B. Donart, E.E. Parker, and J.D. Wallace. 1978. Livestock and vegetational responses to continuous and four-pasture, one-herd grazing system in New Mexico. Proc. First Int. Rangeland Congr. $1: 560-562$.

Pieper, R.D., J.R. Montoya, and V. Groce. 1971. Site characteristics on pinyon-juniper and blue grama ranges in southcentral New Mexico. New Mexico Agr. Exp. Sta. Bull. No. 573.

Price, D.L. 1985. Response of individual plant units to grazing herbivores in three management systems. Ph.D. Thesis. New Mexico State Univ., Las Cruces.

Shephard, H.R. 1971. Effects of clipping on key browse species in southwestern Colorado. State of Colorado Division of Game, Fish and Parks Tech. Pub. 28.

Trlica, M.J., M. Buwal, and J.W. Menke. 1977. Effects of rest following defoliations on the recovery of several range species. J. Range Manage. 30:21-27.

USDC National Oceanic and Atmospheric Administration. 1982. Climatological data, Las Cruces, New Mexico.

Young, V.A., and G.F. Payne. 1948. Utilization of "key" browse species in relation to proper grazing practices in cutover western white pinelands in northern Idaho. J. Forestry 46:35-40. 